\title{
Improved temperature characteristics of laser diodes with nonidentical multiple quantum wells due to temperature-induced carrier redistribution
}

\author{
Ching-Fuh Lin, ${ }^{a}$ Yi-Shin Su, Di-Ku Yu, and Chao-Hsin Wu \\ Graduate Institute of Electro-Optical Engineering and Department of Electrical Engineering, \\ National Taiwan University Taipei, Taiwan, Republic of China \\ Bing-Ruey Wu \\ Telecommunication Lab., Chunghua Telecom Co., Ltd., Yang-Mei, Taoyuan, Taiwan, Republic of China
}

(Received 7 June 2002; accepted 1 April 2003)

\begin{abstract}
Laser diodes with nonidentical multiple quantum wells could have the lasing wavelength very insensitive to temperature variation. For temperature varying from 33 to $260 \mathrm{~K}$, the lasing energy changes less than $5 \mathrm{meV}$, while the band gap energy changes more than $50 \mathrm{meV}$. The origin is due to the strongly temperature-dependent Fermi-Dirac distribution, which favors carriers in high-energy states at large temperature. The temperature-induced carrier redistribution could even cause negative characteristic temperature for a certain temperature range because the low-energy quantum wells behave like reservoirs to overcome the detrimental influence of temperature. (C) 2003 American Institute of Physics. [DOI: 10.1063/1.1577384]
\end{abstract}

Multiple quantum wells (MQWs) have been known for decades to provide many advantages for semiconductor lasers. Those advantages include increasing differential gain, reducing threshold current, increasing characteristic temperature, and so on. However, recent studies have theoretically and experimentally found that carrier distribution in MQWs is not uniform. ${ }^{1-7}$ The nonuniform carrier distribution is influential on the characteristics of quantum-well lasers. ${ }^{5,6,8}$ For semiconductor lasers with identical MQWs, the nonuniform carrier distribution degrades the high-performance of semiconductor lasers, ${ }^{9}$ like causing the recombination loss to increase with injection current. ${ }^{10}$ For semiconductor lasers with nonidentical MQWs, the nonuniform carrier distribution complicates the gain spectrum that is contributed from different types of QWs. ${ }^{11}$ Previous works mostly report that nonuniform carrier distribution degrades the performance of semiconductor lasers. In this work, we discover that nonuniform carrier distribution is not always a detrimental factor. For laser diodes with nonidentical MQWs, the temperaturedependent nonuniform carrier distribution could cause the lasing wavelength to be much less dependent on temperature, compared to the band gap shrinkage. The origin is due to the strongly temperature-dependent Fermi-Dirac distribution, which favors carriers in high-energy states at large temperature. The carrier redistribution then compensates for band gap shrinkage to reduce temperature dependence. Theoretical reasons and experimental details are discussed.

Theoretical explanation of the carrier redistribution resulting from the temperature-dependent Fermi-Dirac distribution is briefly discussed as follows. Figure 1 schematically shows a QW structure that consists of two different QWs. QW A and QW B have the quantized energy level at $E 1$ and $E 2$, respectively. Assume that $E 1$ is much less than $E 2$. As the QW structure is under forward bias, significant carriers are injected into those QWs. The quasi Fermi level is thus

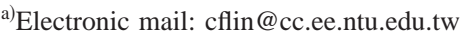

above the quantized levels $E 1$ and $E 2$. The quasi Fermi levels for QW A and QW B are assumed to be $E_{F 1}$ and $E_{F 2}$, respectively, to account for the possible difference of carrier density in the two QWs. On the other hand, the Fermi level usually varies with temperature. However, the variation is small as long as the number of injected carriers is not changed, so the variation is neglected.

The densities of states for QW A and QW B are plotted in Figs. 2(a) and 2(b), respectively. The Fermi-Dirac distributions at two temperatures are also plotted in the same figures. As temperature increases, the Fermi-Dirac distribution changes, causing carriers to move into the high-energy states. As a result, carriers in QW A and QW B change the number of $N 1(R 1-L 1)$ and $N 2(R 2-L 2)$, respectively. $N 1$ and $N 2$ are the density of states for QW A and QW B, respectively. $R 1, L 1, R 2$, and $L 2$ are the shaded areas shown in Fig. 2. For certain injection levels, it is possible that Fermi level $E_{F 1}$ is closer to barrier energy $E_{B}$ than $E 1$ for QW A [Fig. 2(a)], while Fermi level $E_{F 2}$ is closer to $E 2$ than $E_{B}$ for QW B [Fig. 2(b)]. Then the shaded area $R 1$ is less than $L 1$, so the number of carriers in QW A decreases. On the contrary, the shaded area $R 2$ is larger than $L 2$, so the number of carriers in QW B increases. For a fixed injected current level, the total amount of carriers in the QWs is approximately constant. Therefore, as temperature increases, more carriers reside in QW B than in QW A and vice versa as temperature decreases. As a result, under the same injection current, the gain contributed from QW B increases with temperature, while the gain contributed from QW A decreases with tem-

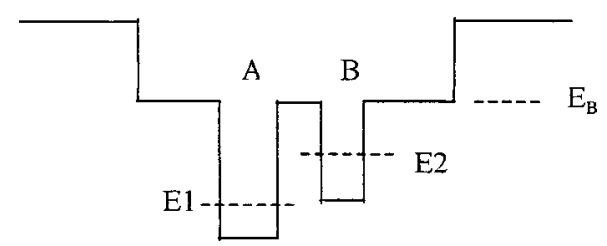

FIG. 1. A schematic of nonidentical MQW structure with two different QWs. 
(a)

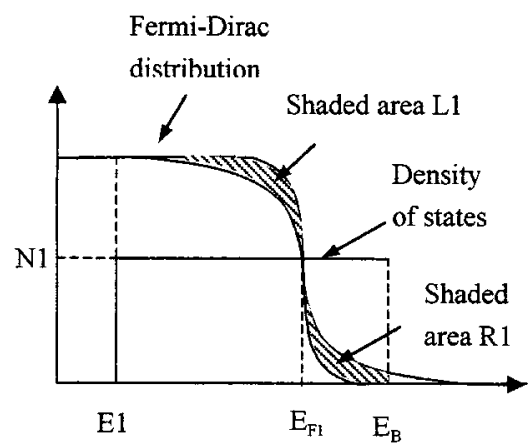

(b)

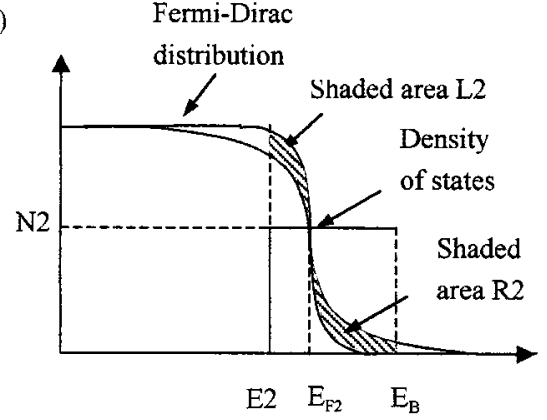

FIG. 2. Variation of Fermi-Dirac distribution at two temperatures for QW A and QW B.

perature. Because the overall gain profile is the overlap of individual gain contributed from each type of QW, the earlier effect will cause the overall gain profile to change its gain peak. This gain peak shifts from energy corresponding to QW A to energy corresponding to QW B as temperature increases. For laser operation, the lasing wavelength usually occurs around the gain peak. Therefore, the lasing wavelength shifts accordingly.

Nonidentical quantum wells with two types of QWs are used for the experiments. The QW structure has two $\mathrm{In}_{0.53} \mathrm{Ga}_{0.47} \mathrm{As}$ QWs near the $p$-cladding layer and three $\mathrm{In}_{0.67} \mathrm{Ga}_{0.33} \mathrm{As}_{0.72} \mathrm{P}_{0.28} \mathrm{QWs}$ near the $n$-cladding layer. The QWs are separated by $15 \mathrm{~nm}$ wide $\mathrm{In}_{0.86} \mathrm{Ga}_{0.14} \mathrm{As}_{0.3} \mathrm{P}_{0.7}$ barriers. The $\mathrm{In}_{0.53} \mathrm{Ga}_{0.47} \mathrm{As}$ QWs and $\mathrm{In}_{0.67} \mathrm{Ga}_{0.33} \mathrm{As}_{0.72} \mathrm{P}_{0.28}$ QWs simulate QW A and QW B of Fig. 1, respectively. At room temperature, their first quantized transition energies are 0.8 and $0.954 \mathrm{eV}$, respectively. Fabry-Pérot laser diodes (LDs) were fabricated on the substrate with the above nonidentical MQWs. The LDs have a ridge waveguide of $5 \mu \mathrm{m}$. Standard processing techniques were used for the fabrication.

The lasing wavelength of LDs is influenced by many factors, but usually occurs around the gain peak that first reaches the loss level. For conventional LDs with identical MQWs, as long as the device length and the injected current level are fixed, the temperature dependence of the gain peak mainly follows the temperature variation of the band gap. Thus, the major influence on the lasing wavelength is band gap shrinkage as temperature increases. For LDs with nonidentical MQWs, temperature variation causes not only the band gap change, but also significant carrier redistribution, as explained previously. The carrier redistribution causes the gain peak of the overall gain profile to shift toward the direction opposite to the band gap variation when temperature changes. Therefore, the measured wavelength of the earlier laser diode has much less temperature dependence, as shown

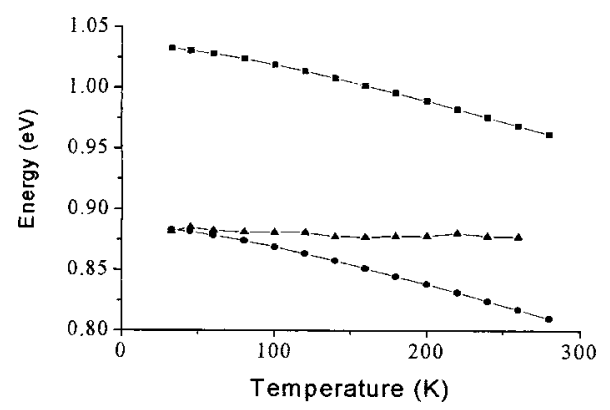

FIG. 3. Variation of transition energy and lasing energy with temperature. (Top curve: $\operatorname{In}_{0.67} \mathrm{Ga}_{0.33} \mathrm{As}_{0.72} \mathrm{P}_{0.28}$ QWs (QW $\mathrm{B}$ ); bottom curve: $\mathrm{In}_{0.53} \mathrm{Ga}_{0.47} \mathrm{As}$ QWs (QW A); middle: oscillation energy of the laser diode with both types of QWs.)

in Fig. 3. The shown energy corresponds to the wavelength of the strongest mode in the measured cluster that consists of several Fabry-Pérot modes. For temperature varying from 33 to $260 \mathrm{~K}$, the corresponding energy of the major mode changes less than $5 \mathrm{meV}$. In contrast, the band gap energy changes more than $50 \mathrm{meV}$ for the same temperature range, as shown by the top and bottom curves in Fig. 3. The reduced temperature variation of lasing wavelength is because carrier distribution favors QW B when temperature increases, causing the lasing wavelength to shift toward the energy corresponding to QW B.

As temperature increases to near room temperature, the threshold and operation current increase. The increased injection level causes more carriers to be captured into the $\mathrm{In}_{0.67} \mathrm{Ga}_{0.33} \mathrm{As}_{0.72} \mathrm{P}_{0.28}$ QWs. ${ }^{11}$ Because both the temperature and the increased carrier injection favor carriers to reside in the $\mathrm{In}_{0.67} \mathrm{Ga}_{0.33} \mathrm{As}_{0.72} \mathrm{P}_{0.28} \mathrm{QWs}$, such QWs contribute more gain to lasing modes. Therefore, another cluster of FabryPérot modes at short wavelength appears. Figure 4 shows the lasing spectrum with two clusters of lasing modes at $29^{\circ} \mathrm{C}$. The inset is the variation of lasing wavelength versus temperature. The significant increase of carriers in the $\mathrm{In}_{0.67} \mathrm{Ga}_{0.33} \mathrm{As}_{0.72} \mathrm{P}_{0.28} \mathrm{QWs}$ causes the original cluster of lasing modes at long wavelength to be blueshifted as temperature increases.

Because the two clusters of lasing wavelengths are widely separated spectrally, it is easy to measure their lasing powers separately. We discover that as temperature increases, the lasing power of the short-wavelength modes increases, while long-wavelength modes have decreasing lasing power. This further indicates that the gain contributed from the $\mathrm{In}_{0.67} \mathrm{Ga}_{0.33} \mathrm{As}_{0.72} \mathrm{P}_{0.28} \mathrm{QWs}$ increases, while the gain contributed from the $\mathrm{In}_{0.53} \mathrm{Ga}_{0.47} \mathrm{As}$ QWs decreases. The reason is

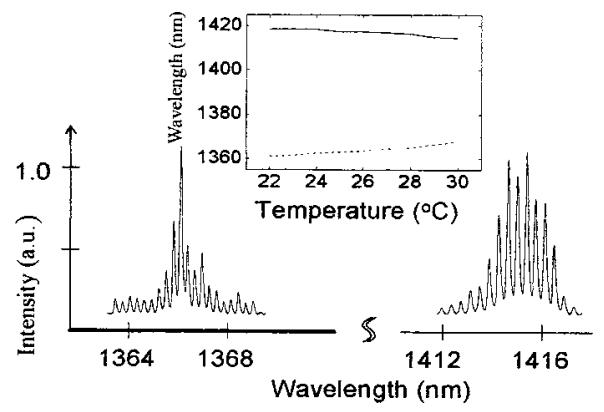

FIG. 4. Emission spectrum with two clusters of lasing modes. (Inset is the variation of the lasing wavelength vs temperature.) 


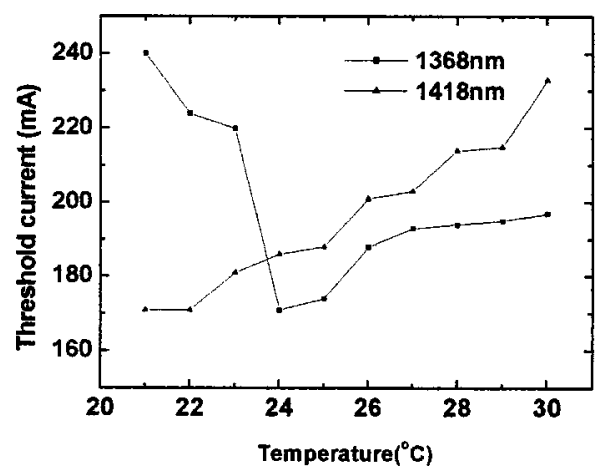

FIG. 5. Variation of threshold current of long-wavelength and shortwavelength modes with temperature.

because the number of carriers in the $\operatorname{In}_{0.67} \mathrm{Ga}_{0.33} \mathrm{As}_{0.72} \mathrm{P}_{0.28}$ QWs increases, while the number of carriers in the $\mathrm{In}_{0.53} \mathrm{Ga}_{0.47}$ As QWs decreases when temperature increases. In addition, the lasing threshold of the short-wavelength modes becomes less than that of the long-wavelength modes for temperature beyond $24^{\circ} \mathrm{C}$, as shown in Fig. 5. Furthermore, in the temperature range between 21 and $24^{\circ} \mathrm{C}$, the threshold current for the short-wavelength modes decreases, implying that those modes have negative characteristic temperature. In the past, the laser diodes usually have positive characteristic temperature. Such an observation of negative characteristic temperature shows the possibility of using carrier distribution among nonidentical MQWs to override bad effects like carrier leakage or increased Auger recombination caused by temperature increase.

Nonunifrom carrier distribution has been found to exist in MQWs. ${ }^{1-7}$ Our investigation further indicates that nonuniform carrier distribution is also strongly influenced by temperature due to the nature of Fermi-Dirac distribution. The temperature effect on the nonuniform carrier distribution may overcome other detrimental influences of temperature.

In conclusion, the strongly temperature-dependent Fermi-Dirac distribution favors carriers to reside in highenergy states, causing the gain in QWs of low energy to decrease and the gain in QWs of high energy to increase. As a result, laser diodes with nonidentical MQWs have their lasing wavelength much less dependent on the temperature variation. For temperature varying from 33 to $260 \mathrm{~K}$, the energy of the lasing mode changes less than $5 \mathrm{meV}$, while the band gap energy changes more than $50 \mathrm{meV}$. In addition, the temperature-induced carrier redistribution could even result in negative characteristic temperature for a certain temperature range.

This work is supported in part by National Science Council, Taipei, Taiwan, R.O.C. under Contract No. NSC902215-E-002-016.

${ }^{1}$ R. Nagarajan, T. Fukushima, S. W. Corzine, and J. E. Bowers, Appl. Phys. Lett. 59, 1835 (1991).

${ }^{2}$ A. Hangleiter, A. Grabmaier, and G. Fuchs, Appl. Phys. Lett. 62, 2316 (1993).

${ }^{3}$ N. Tessler and G. Eisenstein, IEEE J. Quantum Electron. 29, 1586 (1993).

${ }^{4}$ K. Frojdh, S. Marcinkevicius, U. Olin, C. Silfvenius, B. Stalnacke, and G. Landgren, Appl. Phys. Lett. 69, 3695 (1996).

${ }^{5}$ H. Yamazaki, A. Tomita, and M. Yamaguchi, Appl. Phys. Lett. 71, 767 (1997).

${ }^{6}$ B.-L. Lee, C.-F. Lin, J.-W. Lai, and W. Lin, Electron. Lett. 34, 1230 (1998).

${ }^{7}$ M. J. Hamp, D. T. Cassidy, B. J. Robinson, Q. C. Zhao, D. A. Thompson, and M. Davies, IEEE Photonics Technol. Lett. 10, 1380 (1998).

${ }^{8}$ M. J. Hamp, D. T. Cassidy, B. J. Robinson, Q. C. Zhao, and D. A. Thompson, Appl. Phys. Lett. 74, 744 (1999).

${ }^{9}$ D. Ban and E. H. Sargent, IEEE J. Quantum Electron. 36, 1081 (2000).

${ }^{10}$ J. Piprek, P. Abraham, and J. E. Bowers, IEEE 16th Int. Semiconductor Laser Conf., 1998, p. 167.

${ }^{11}$ C.-F. Lin, B.-R. Wu, L.-W. Laih, and T.-T. Shih, Opt. Lett. 26, 1099 (2001). 
Applied Physics Letters is copyrighted by the American Institute of Physics (AIP). Redistribution of journal material is subject to the AIP online journal license and/or AIP copyright. For more information, see http:/ojps.aip.org/aplo/aplcr.jsp

Copyright of Applied Physics Letters is the property of American Institute of Physics and its content may not be copied or emailed to multiple sites or posted to a listserv without the copyright holder's express written permission. However, users may print, download, or email articles for individual use. 